\title{
Experimental and theoretical investigation of the fracture behavior of glass beads/epoxy composites using microscratching
}

\author{
SHAHLAA A. AL WAKEEL ${ }^{*}$, MIJA H. HUBLER ${ }^{\dagger}$ \\ *University of Colorado Boulder \\ Boulder, CO USA \\ e-mail: shahlaa.alwakeel@colorado.edu \\ ${ }^{\dagger}$ University of Colorado Boulder \\ Boulder, CO USA \\ e-mail : hubler@colorado.edu
}

Key words: Fracture toughness, Scratch testing, Composite materials

\begin{abstract}
Concrete, ceramic, and other quasi-brittle materials contain pre-existing cracks and complex structure on various internal length-scales. Quantifying the fracture behavior of these heterogeneous materials using different testing methods is a challenge and is an argumentative subject [1]. To selectively study the impact of microstructure on micro-scale fracture parameter this article presents an experimental and theoretical investigation of the fracture toughness of a representative composite material consisting of glass beads embedded in epoxy using microscratching and conventional three-point bending tests. Existing analysis formulas for microscratch data assumes homogeneity near the indenter tip. This study finds that this assumption may need to be reconsidered. By controlling the volume fraction of glass beads (representing an inclusion) in the matrix of the composite material, it is possible to control the amount, or direction, of crack development and reduce the stress concentration within the material during loading ${ }^{[1]}$. As a result, an increase in the fracture toughness of the composites and improved fracture properties is obtained.
\end{abstract}

\section{INTRODUCTION}

Indenter-based fracture testing techniques have had a broad application in characterizing material resistance to fracture propagation for over thirty years. The microscratch test has been used to determine the fracture toughness and the penetration depth of composite materials ${ }^{[1]}$. Akono et al. used the scratch test to measure forces and penetration depth by dragging an indenter probe along the surface material. This technique was successfully applied to several materials ranging from polymers to metals ${ }^{[2]}$. However, the formulation for energy release due to scratching applies the $J$-integral in front of the crack tip, and in doing so assumes a homogenous stress field in front of any crack tip that is generated. This ignores the local effect of the inclusions on this stress field. This paper tests the validity of this assumption.

The effect of inclusions on fracture parameters is known. Erwin et al. studied the effect of increasing the filler content on the composite modulus. It has been found that the modulus and fracture parameters are improved with increased volume fraction of the filler ${ }^{[3]}$. However, the quantification of the relevant microstructure in concrete introduces many experimental uncertainties, so the present investigation uses a model system to capture the relevant mechanics. Many researchers have used a similar model composite system formed from epoxy resins filled with glass beads to evaluate the mechanical properties in terms of stress intensity fracture $\left(K_{I}\right)$ and the energy release rate $\left(G_{I}\right)^{[1,4]}$. The aim of this research is to 
analytically and experimentally investigate the effect of the glass beads (inclusion) content in the matrix on the fracture properties of the composite material by using the microscratch test. Understanding this would allow us to further pursue the questions: How can we improve the microscratch analysis methods? Is it possible to control the amount, or direction, of crack development by controlling the spatial distribution of the glass beads in the matrix?

\section{EXPERIMENTAL WORK}

\subsection{Raw materials and composites preparation}

For all the specimens hollow microspheres, namely $3 \mathrm{M}$ Scotchlite ${ }^{\mathrm{TM}}$ glass beads (soda-lime) S38 microspheres were used as inclusions. The epoxies used as the binder consisted of the solid resin DGEBA (diglycidyl ether of bisphenol A) from Dow chemical Co. and the hardener DDS (4,4'-diaminodiphenylsulphone) from Sigma Aldrich Chemical Company. The physical properties of the epoxy "binder" and the glass beads "inclusion" are listed in Table 1. The fabrication of the composites were carried out using the following steps: (i) according to Ref. ${ }^{[4}$, ${ }^{5]}$ the epoxy DER 661 was melted for an hour (ii) Then the glass beads were mixed with different volume percentage ranging from $0 \%$ to $50 \%$ at a time (iii) The mixture was combined with stoichiometric amounts of the curing agent DDS (98\%) for thirty minutes (iv) once uniform the mixtures were cast in a pre-heated mold (v) finally, the specimen were placed in a convection oven for sixteen hours at $160^{\circ} \mathrm{C}$ till the temperature increased to $200^{\circ} \mathrm{C}$ for two hours. Then the specimen was allowed to cool slowly in the oven to room temperature.

Table 1: Physical properties of the epoxy and the glass beads

\begin{tabular}{lcc}
\hline Material type & $\begin{array}{c}\text { True density } \\
\text { (g/cc) }\end{array}$ & $\begin{array}{c}\text { Young's Modulus } \\
(\mathrm{GPa})\end{array}$ \\
\hline DER661/DDS & 1.204 & 2.8 \\
\hline Glass beads S38 & 0.38 & 70 \\
\hline
\end{tabular}

\subsection{Micro Scratch Test}

A microscratch tester with a capacity of 200N from Anton Paar was used to introduce a scratch in the specimen surface with a $200 \mu \mathrm{m}$ Rockwell diamond indenter. Panoramic pictures of the surface were taken prior to performing any scratch as shown in Figure 1. The scratch test was performed in three stages staring with a prescan phase in which the specimen surface was investigated by the indenter tip with a minimum load of $0.05 \mathrm{~N}$.

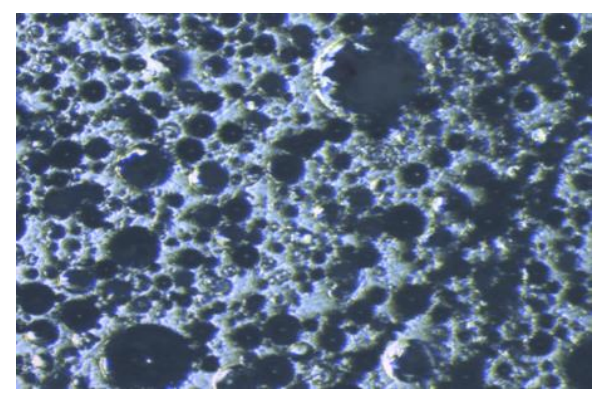

Figure 1: Typical SEM image of surface composites for $50 \%$ specimen. The different circular inclusions represent sections of the spherical glass beads which have been cut and polished down at different height.

During the second stage a scratch is introduced on the specimen's surface. At the same time the vertical and the horizontal forces as well as the penetration depth were recorded as the probe is pushing into the material at depth $\mathrm{d}$. The scratch is produced by the projected horizontal load bearing contact area not by the probe tip as shown in Figure 2. The scratch testing parameters used in this test are listed in Table 2.

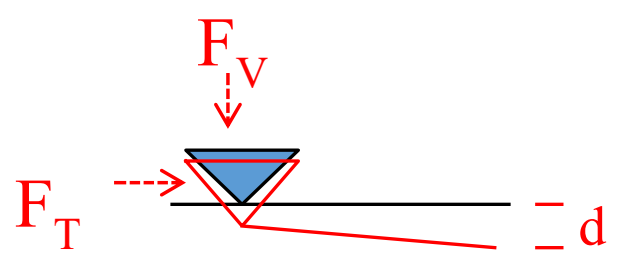

Figure 2: Schematic of scratch test. 
Table 2: Testing parameters used during the scratching test

\begin{tabular}{ll}
\hline Scratch test parameters & \\
\hline Minimum vertical load $(\mathrm{N})$ & 0.05 \\
\hline Maximum vertical load $(\mathrm{N})$ & 0.5 \\
\hline Loading rate $(\mathrm{N} / \mathrm{min})$ & 0.9 \\
\hline Speed $(\mathrm{mm} / \mathrm{min})$ & 10 \\
\hline Scratch length $(\mathrm{mm})$ & 5 \\
\hline
\end{tabular}

The scratch probe parameters used in this test are listed in Table 3.

Table 3: Scratch probe parameters

\begin{tabular}{ll}
\hline Indenter parameters & \\
\hline Type & Rockwell \\
\hline Material & Diamond \\
\hline Radius $(\mu \mathrm{m})$ & 200 \\
\hline
\end{tabular}

The last stage is the "panorama view phase", in order to image the physical crack a series of panoramic pictures have been taken for whole scratching process to image the physical cracks on the residual groove after the damage or the cracking.

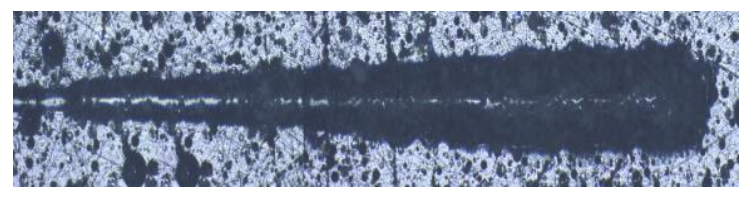

Figure 3: Panorama Image of a scratch for the $50 \%$ inclusion specimen

The fracture toughness in terms of $\left(K_{C}\right)$, the stress intensity factor was calculated from the horizontal scratching force $\mathrm{F}_{\mathrm{T}}$, and the projected load bearing contact area $\mathrm{A}$ and the perimeter $p$ according to the relationship developed in Akono et al ${ }^{[1]}$.

$$
K_{C}=\frac{F_{T}}{\sqrt{2 p A}}
$$

where

$F_{T}=$ the horizontal force

$p=$ the scratch probe perimeter

$A=$ the horizontal projected load bearing contact area.

\subsection{Scanning of specimen surface}

Before running the scratch test, the specimen's surface roughness was scanned and imaged for analysis. The Figures below represent the Image segmentation by global threshold using Otsu's algorithm ${ }^{[6]}$. The image was displayed using the level (0.6078) in white and back colors. The spatial statistics were used to check for the quality of the inclusion arrangement.

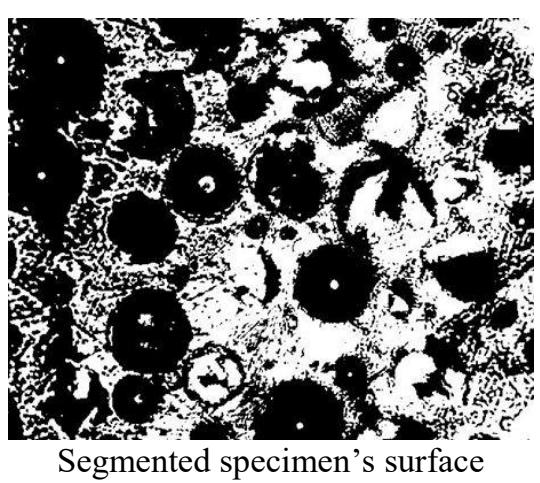

Figure 4: Converting the scanned surface pictures to black and white color using Otsu's algorithm for 50\% glass beads

\subsection{The ASTM Standard three-point test}

Three point bending tests on single-edgenotched specimens were conducted to determine the critical stress intensity factor $K_{C}$ using standard methods for comparison. The specimen thickness was selected to satisfy the plane strain requirements ASTM $3999^{[7]}$ section 7. The specimen's dimensions that will be used in this study are the same as the specimen's dimensions used by Lee ${ }^{[3]}$. Per ASTM 399 section 7.2.1.1, the width of the specimen $\mathrm{W}=2 *$ thickness $\mathrm{B}$. The thickness used in this study is $B=6.75 \mathrm{~mm}$ and the width is $\mathrm{W}=12.7 \mathrm{~mm}$. The crack length $\mathrm{a}$, is equal to the thickness $\mathrm{B}^{[4]}$. The support span length is $S=50.8 \mathrm{~mm}$. By inserting a razor blade into the specimen, a notch was created in the specimen, Figure 5 illustrate the specimen dimensions and notch shape.

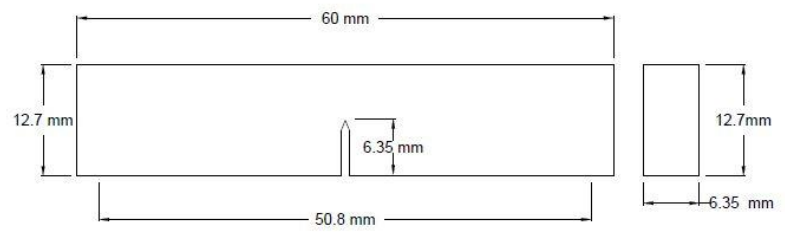

(a) 


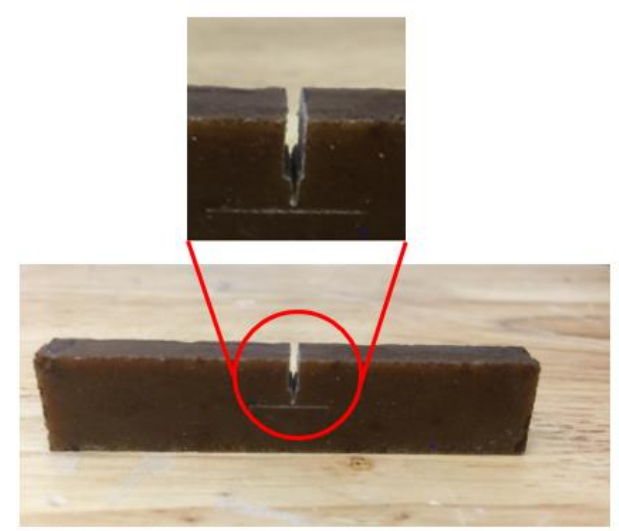

(b)

Figure 5: (a) Schematic representation of specimen geometry used in this study; (b) $5 \%$ glass beads specimen

The load is applied by means of a constant displacement at a cross head speed of 2.54 $\mathrm{mm} / \mathrm{min}$. The stress intensity factor was calculated using the following equation:

$$
K_{C}=f\left(\frac{a}{w}\right) \frac{3 P S \sqrt{a}}{2 B W^{2}}
$$

where:

$\mathrm{K}_{\mathrm{C}}=$ Critical stress intensity factor

$\mathrm{P}=$ load at failure

$\mathrm{S}=$ Span length $=60 \mathrm{~mm}$

$\mathrm{a}=$ Crack length $=6.35 \mathrm{~mm}$

$\mathrm{W}=$ Specimen width $=12.7 \mathrm{~mm}$

$\mathrm{f}\left(\frac{a}{w}\right)=$ shape factor is calculated by using the equation below:

$f\left(\frac{a}{w}\right)=1.93-3.07\left(\frac{a}{w}\right)+14.53\left(\frac{a}{w}\right)^{2}-$

$25.11 *\left(\frac{a}{w}\right)^{3}+25.80\left(\frac{a}{w}\right)^{4}$

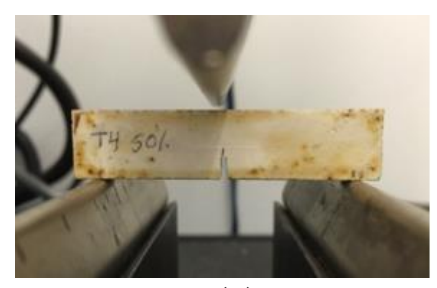

(a)

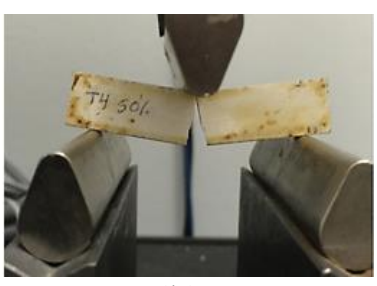

(b)
Figure 6: Three-point test for $50 \%$ glass beads (a) before test; (b) after test

\section{RESULTS AND DISCUSSION}

Modeling and testing was performed on resin composites with four different volume fractures of microspheres ranging from $0 \%, 5 \%, 25 \%$ and $50 \%$ to study the influence of the microstructure inclusion content on the fracture parameters of the composite material. A comparison with the observed trend was then investigated using conventional three-point bending test on precracked specimens.

\subsection{Fracture toughness using Micro Scratch Test}

Figures 7-10 displays the relation between the vertical force and the penetration depth for the four specimens considered in this research. Some scratch curves have kink points where they transition from a smooth parabolic curve to a noisy linear trend. In particular, those points are occurring in the specimens containing a $5 \%$ vol. fraction of glass beads.

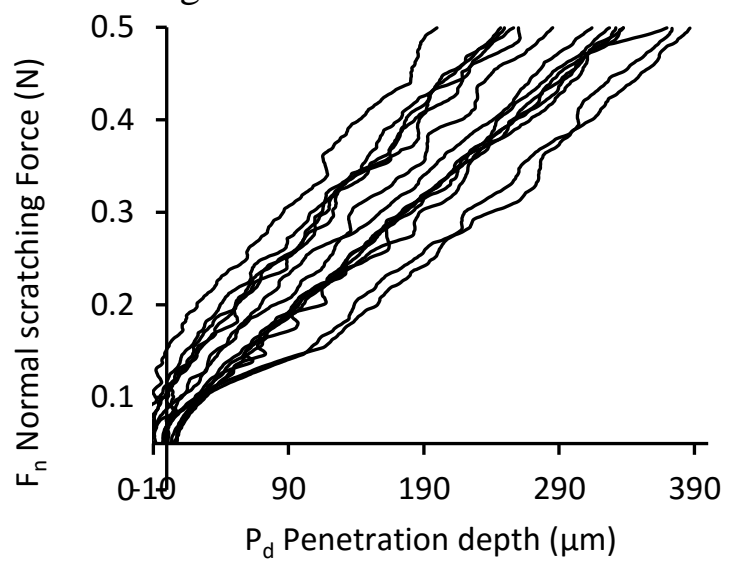

Figure 7: The relation between the normal scratching force and the penetration depth for $50 \%$ specimen.

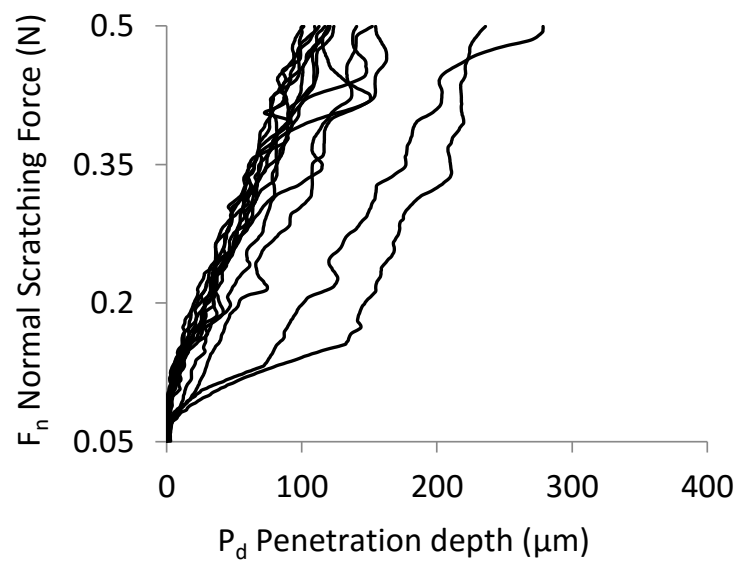

Figure 8: The relation between the normal scratching force and the penetration depth for $25 \%$ specimen 


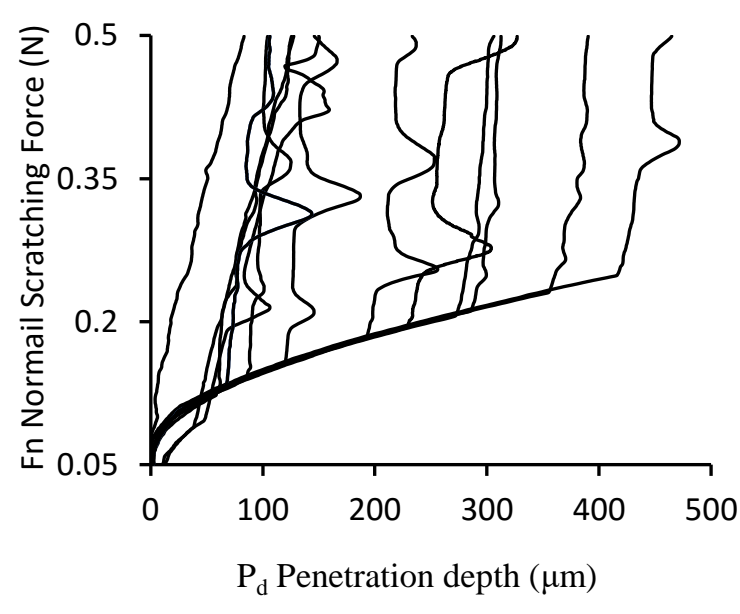

Figure 9: The relation between the normal scratching force and the penetration depth for $5 \%$ specimen

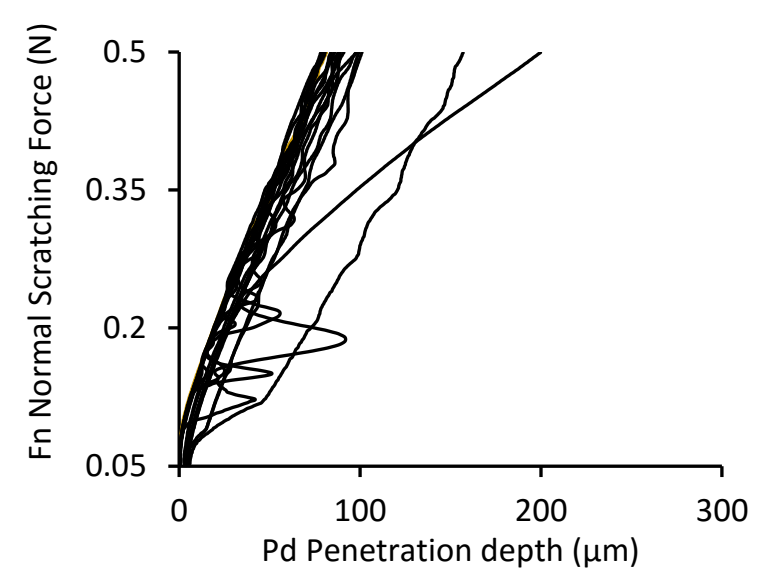

Figure 10: The relation between the normal scratching force and the penetration depth for $0 \%$ specimen.

Figure 11 shows the variation of the fracture toughness with the glass bead content for the scratches with and without kink points. Between the $0 \%$ and $5 \%$ there is an increase in the fracture toughness value due to toughening mechanisms as the filler content increases. Beyond the 5\% glass beads content the fracture toughness decreases due to microspheres de-bonding from the matrix. The de-bonding is accompanied by micro-cracks. If the directions of these cracks are parallel to the crack growth direction this would facilitate the crack propagation. Similar trends were found by Erwin ${ }^{[4]}$. The most distinctive feature in Figure 11 is the significant decrease in the fracture toughness for inclusion content up to $50 \%$ comparing to the neat epoxy resin. The reason behind that is the toughening mechanism is changing as the inclusion content is increased, this is caused by spacing theory between particle spacing as mentioned by Lee and Yee ${ }^{[3]}$ for glass beads embedded in epoxy resin composites. In this figure we can notice that at $5 \%$ glass bead content the fracture toughness value is more scattered than for the other composites.

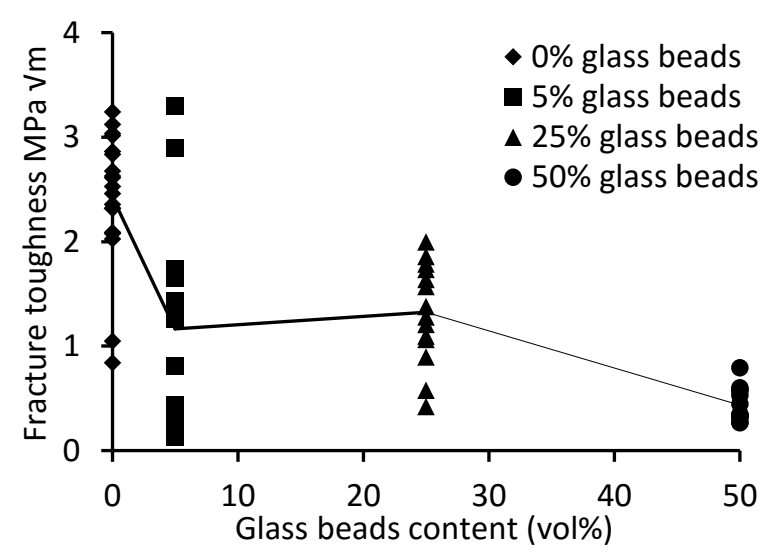

Figure 11: Fracture toughness trend for different glass bead contents using the scratch test

\subsection{Fracture toughness from 3PB test}

A conventional three-point bending test with single edge notched geometry was used to determine the material fracture toughness again. The variation of the fracture toughness with the glass beads content for the three-point test is shown in Figure 12.

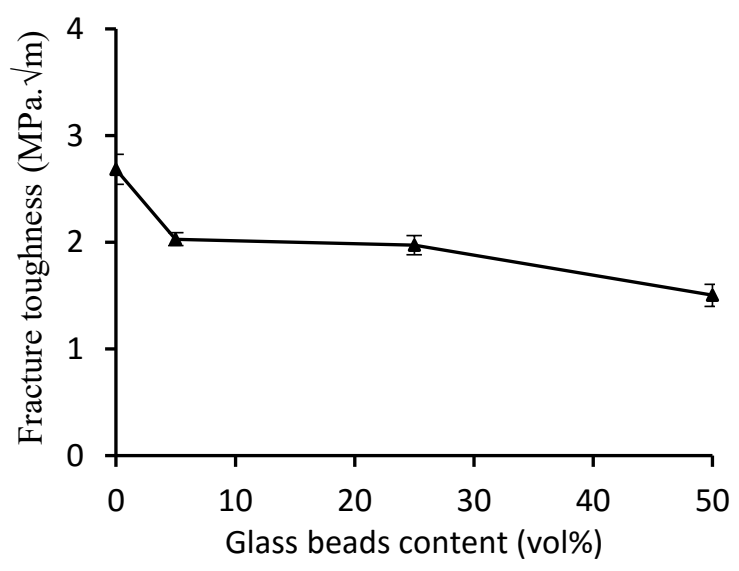

Figure 12: Fracture toughness trend for different glass bead contents using the three-point bend test

A large discrepancy was found between the data sets shown in Figure 11 and 12. The curve has the same trend; however, different fracture 
values. This discrepancy could be from the effect of microstructural defects and system arrangement, which is detected by the microscratch test by pushing the probe along of the specimen surface. Since it is also present in the zero inclusion case it may derive from the difference between the different natures of the two tests. In the case of the three-point test we load the material until the failure and allow crack stresses to redistribute loading throughout the specimen while in the microscratch test we regulate the loading locally and do not allow the whole sample structure to assist in redistributing the applied load. As a result, local material crushing phenomena are much more prevalent in the scratch test than three-point bending tests and we do not allow the structure to act as a whole. In this paper we will investigate the effect of the microstructure and leave the second hypotheses for future work.

\subsection{Investigating the microstructure}

The individual scratch test measurements for different inclusions content was plotted in Figure 13. The increase in inclusion density as in curves (c) and (d) results in uniform and homogenous material behavior, no kink or scatter in the plotted curves. There is a similar trend in the pure epoxy as indicated in curve (a). While a different trend can be noticed in (b), this implies that, in low concentration of the inclusion the behavior is changed because of the heterogeneous materials during the scratch test. We will further investigate this theory using modeling methods.

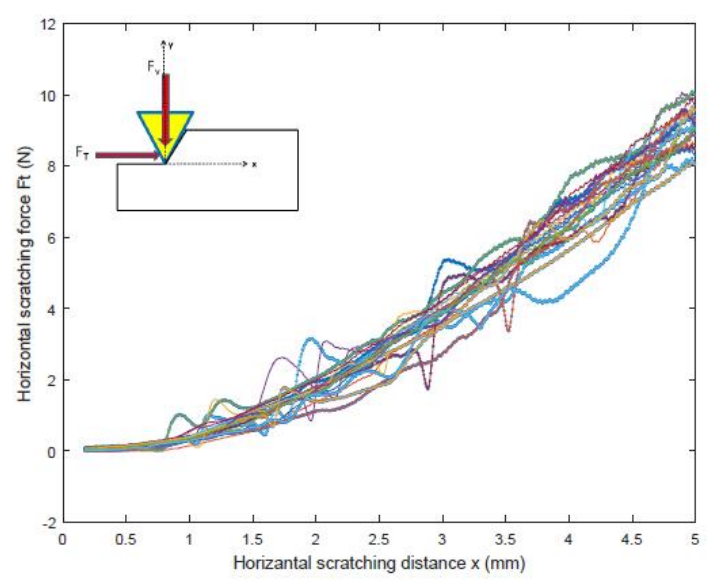

(a)

$0 \%$ Glass beads

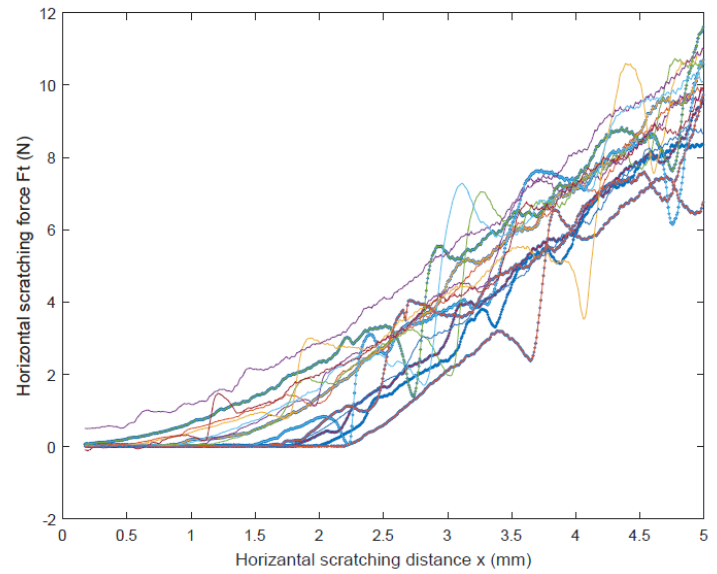

(b)

$5 \%$ Glass beads

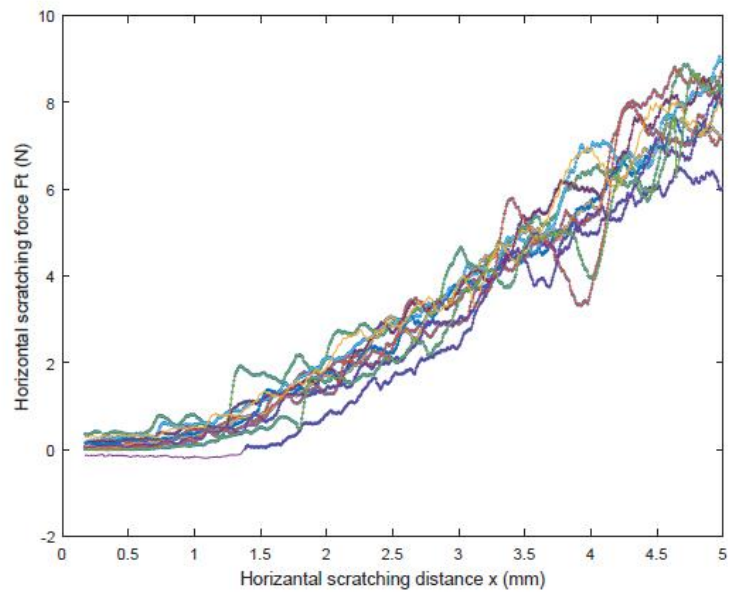

(c)

$25 \%$ Glass beads

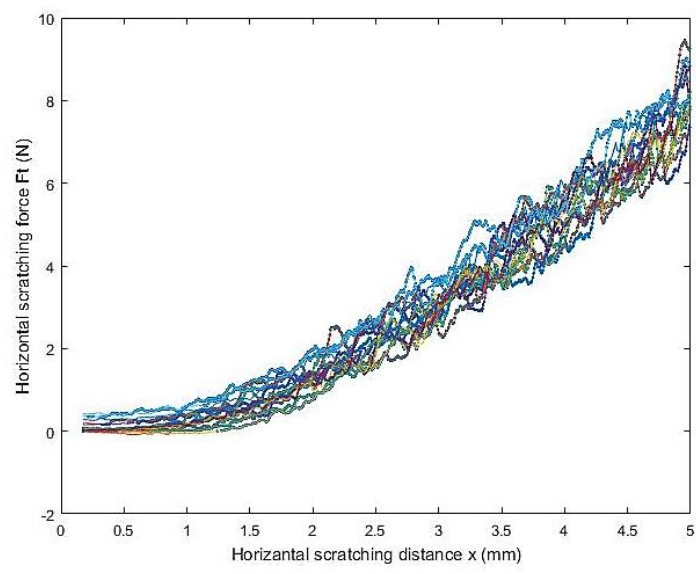

(d)

$50 \%$ Glass beads

Figure13: Horizontal scratching force versus horizontal distance for different glass bead contents

By augmenting linear elastic fracture mechanics analysis of the scratch data, the fracture toughness for different compositions of the glass beads embedded in epoxy resin was 
derived. The fracture toughness, $K_{c}$ for different compositions of the glass beads embedded in epoxy resin is presented in Figure 11. When considering the scatter in in the results, no clear overarching trend is observed. To test our hypothesis that the kink observed in some of the scratch data is due to the presence of the inclusions, we separated the data affected by the kink in Figure 11. After this separation, two clear trends are observed in fracture response. Thus we propose that the kink is due to a change in boundary conditions when an inclusion is near the indentor tip. To confirm that such a change in boundary conditions could generate this effect, we will formulate a mathematical model considering this change and study the effect of probability of finding such an inclusion near the probe tip.

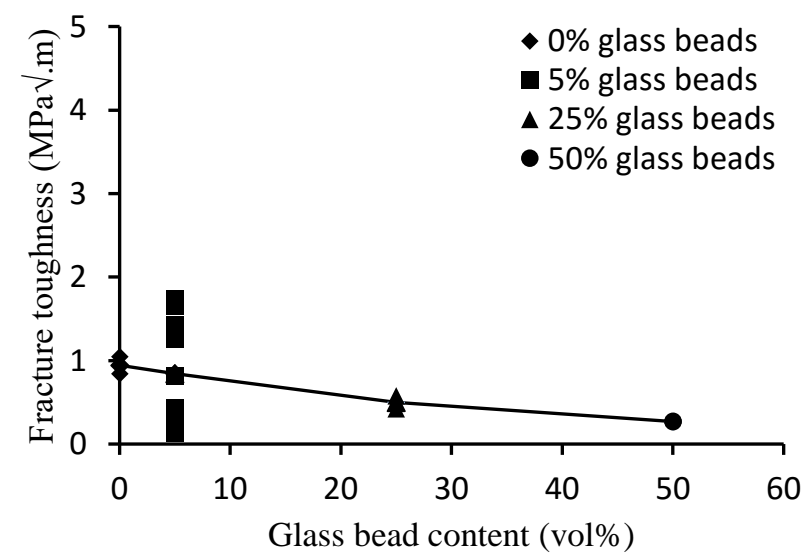

(a)

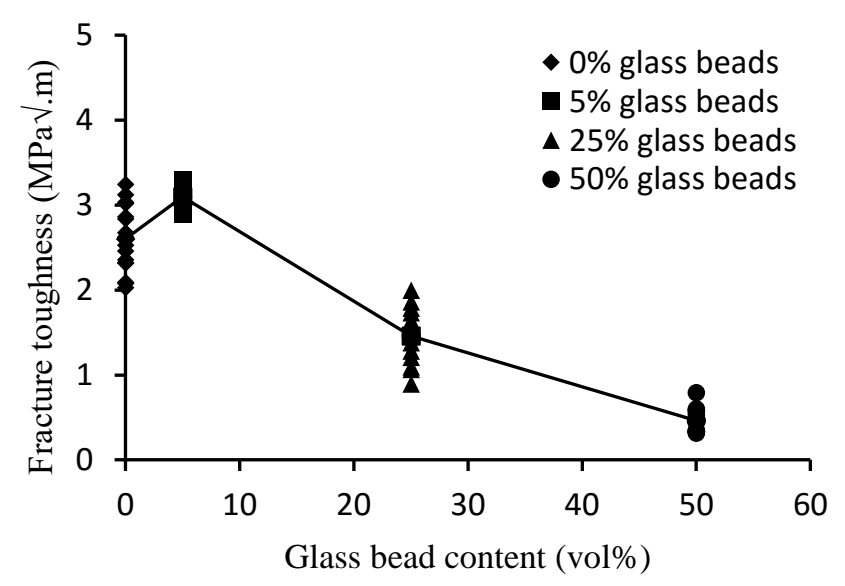

(b)

Figure 14: (a) Fracture toughness range for different glass bead content with kink points (b) Fracture toughness range for different glass bead contents without kink points

\subsection{Experimental and Numerical confirmation that kink curves capture inclusions}

As has been shown in the Figure 14 (a) the shape of the fracture energy as a function of inclusion volume is captured due to a change in the microstructure of the material near the indenter tip. This only holds true for the curves showing a kink, which is hypothesized to relate the presence of an inclusion in front of the crack tip. For the purpose of comparison between the behavior using different techniques we show this curve again in Figure 16 (a) with average values for each percent.

In order to confirm the effect of microstructure on the material behavior, a numerical investigation is presented using finite element analysis of a centrally cracked composite material containing a single inclusion located near the crack tip. The fracture toughness is determined using the displacement extrapolation method, which uses nodal displacements near the crack tip. The analysis in this research is carried on microstructure level, therefore the fracture toughness is calculated based on the Linear Elastic Fracture Mechanics assumption and the concepts of plane strain conditions for mode I failure. The element used in this analysis is two dimensional with eight or six nodes having two degrees of freedom at each node. The deformed configuration of the specimen used in this simulation is shown in Figure 15.

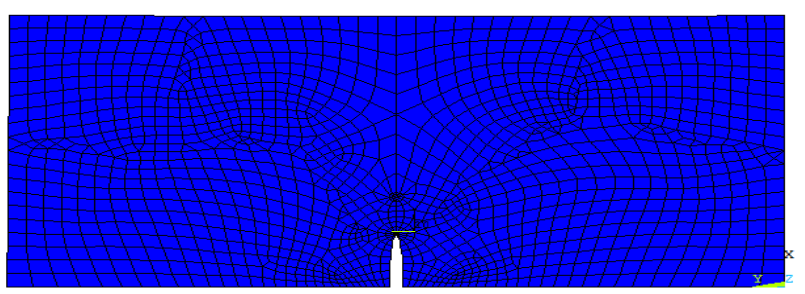

Figure 15: The deformed configuration of the specimen

A series of numerical simulations was conducted selecting different inclusion Young's moduli $\left(E_{i}\right)$ for the embedded inclusion in front of the crack tip to evaluate the relation between the normalized fracture toughness $\left(K_{I} / K_{0}\right)$ and the normalized modulus of elasticity $\left(E_{i} / E_{0}\right)$. Where $E_{0}$ and $E_{i}$ represent the Young's modulus 
for matrix and inclusion respectively, $K_{0}$ and $K_{I}$ are the fracture toughness without and with inclusion respectively. The results are presented in Figure 16 (b), as the value of the inclusion stiffness increases the stress intensity factor value decreases.

A good agreement was found between the results of this analysis as presented in Figure 16 $(\mathrm{a} \& \mathrm{~b})$ and the experimental work presented by Papaioannou et al ${ }^{[8]}$ as shown in Figure 16 (c). The same trend found from the theory for various ratios of Young's modulus $\left(\mathrm{E}_{\mathrm{i}} / \mathrm{E}_{0}\right)$ presented by Zhonghua et al ${ }^{[8]}$ as illustrated in the Figure 16 (d).

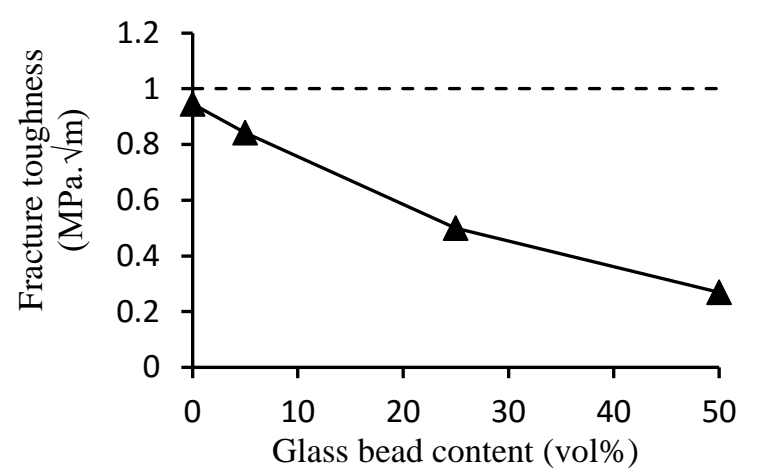

(a)

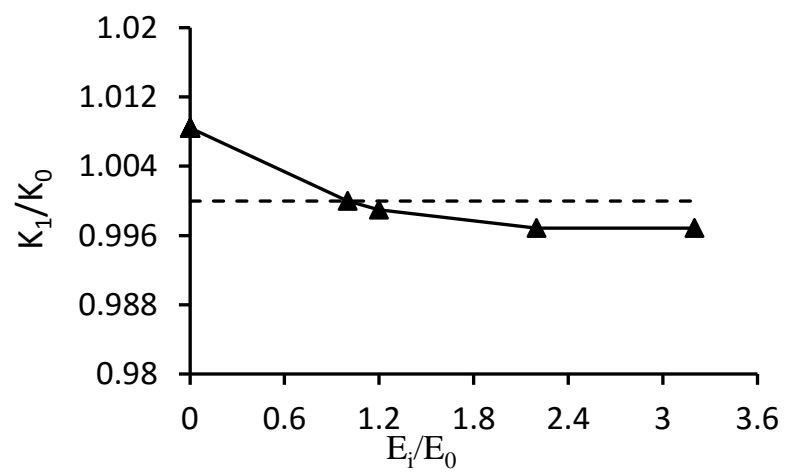

(b)

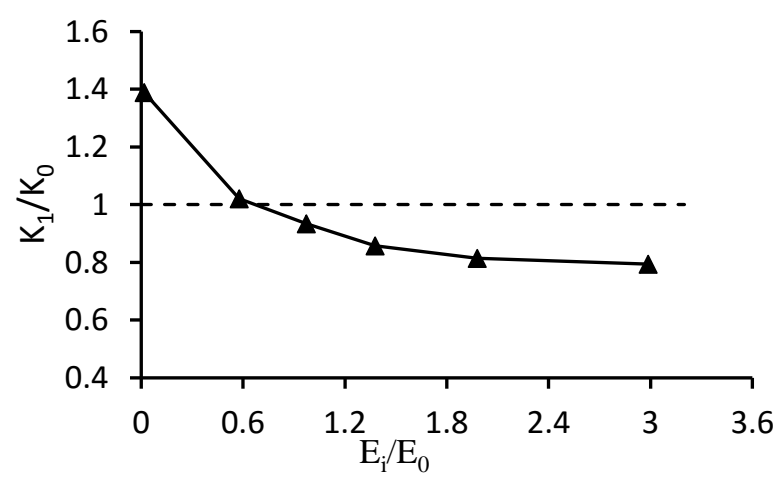

(c)

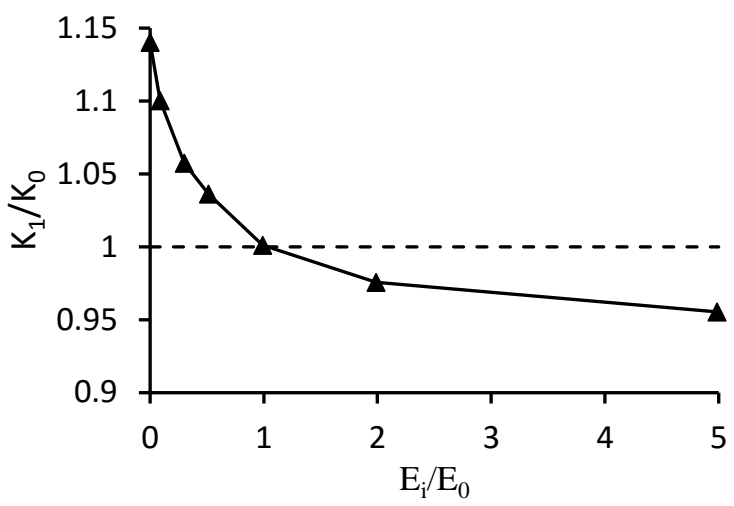

(d)

Figure 16: Comparison between numerical and theoretical formulations behavior of crack-inclusion interaction within crack tip field for mode I failure. The fracture toughness has different values based on different materials:

(a) The average values of the fracture toughness from the scratch test for the curves showing a kink-current work.

(b) Fracture toughness from numerical simulation-current work.

(c) Numerical formulation for central cracked specimen by Papaioannou et al ${ }^{[8]}$.

(d) Theoretical formulation for the impact of single inclusion located ahead of crack tip in 3PB specimen by Li et al ${ }^{[8]}$.

\subsection{Mathematical simulation}

We formulated the linear viscous behavior of glass beads embedded in epoxy resin composites during scratch testing. The standard linear solid (Zener model) was used to interpret the experimental results data and predicate the behavior of the composite.

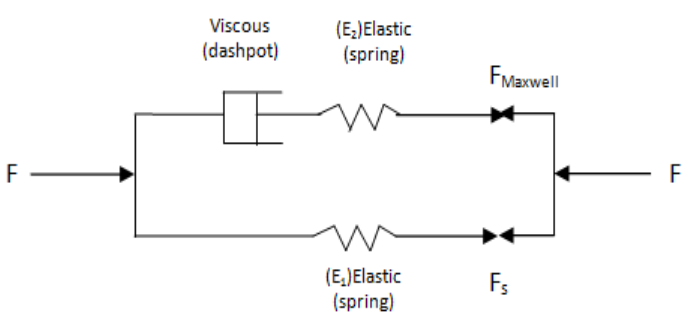

Figure 17: Standard linear solid (Zener model)

The following assumptions are made in modeling the behavior of the polymer:

1. The crack is a straight line and propagates along the $\mathrm{x}$-axis.

2. A two-dimensional representation of the model will be considered.

3. The Cartesian coordinates will be used throughout the derivation. 


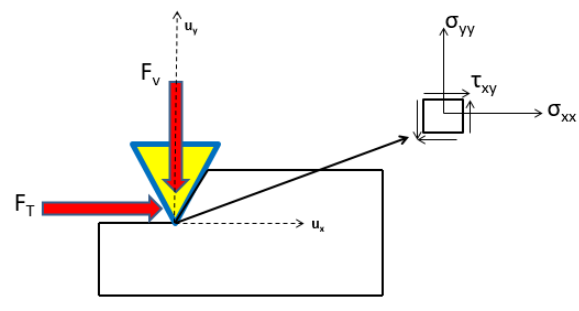

(a)

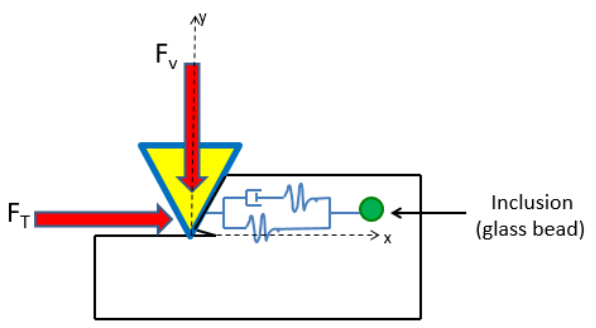

(b)

Figure18: (a) Stress and displacement components in the Cartesian coordinate system

(b) Using standard linear solid model to model the material behavior

Formulation of visco-elastic stress strain constitutive relation of the material to mathematically simulate the behavior of a material using the standard linear solid model illustrated in the Figure 18. The following constitutive equation has been used to relate the strain to the stress.

$$
\varepsilon_{t}=\frac{\sigma_{0}}{\left(E_{1}+E_{2}\right)}\left[1-e^{-\frac{\left(E_{1}+E_{2}\right) t}{\eta}}\right]
$$

When the spring is connected to dashpot in series yields a Maxwell model. A combination of the Maxwell model in parallel with a spring as its illustrated in the figure below yields the model that we proposed for in this study to predict the shape of the compressive stress-strain curve ${ }^{[9]}$. If an inclusion is found far from the scratch probe the material is best represented with moduli of the springs in the Zener model being identical, while very stiff close inclusions will require unequal moduli. The observed transition point in the material response can be captured with this material model by changing the material stiffness to represent the various distances to the nearest inclusion as shown in Figure 19.

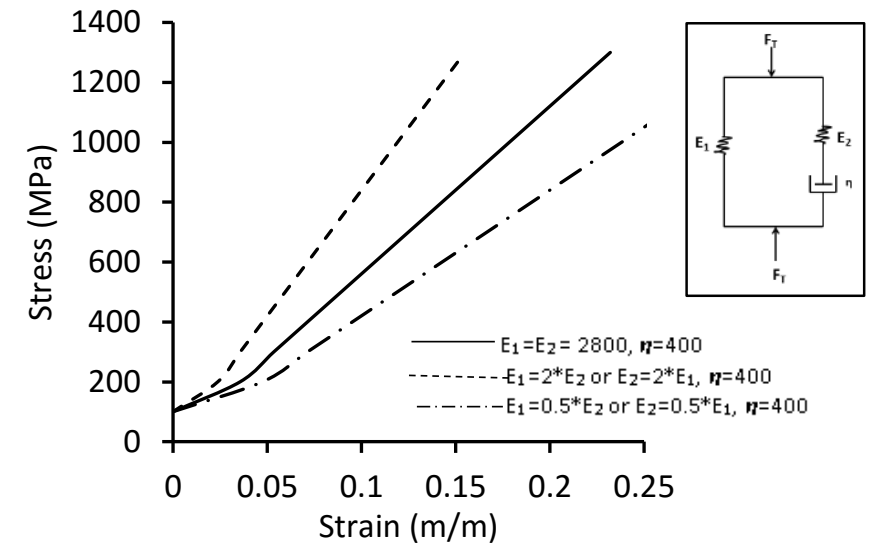

Figure 19: Compression stress-strain curve describes by the model on the right side of the figure

\subsection{Random probability distribution}

Random probability distributions were generated to test if the increase in noise is also due to the arrangement of the inclusions. Three different populations of random probability distribution of particles (pixels) were generated to calculate $\mathrm{g}(\mathrm{r})$, the radial distribution function. This represents the probability of finding a nearby inclusion at any location in the sample. A random placement of inclusions was generated using MATLAB to create sixty different fields of distributions of random pixels for each population. The populations were chosen to match the experimental test ranging from $5 \%$, $25 \%$ and $50 \%$. Figure 18 illustrate the results of this analysis.

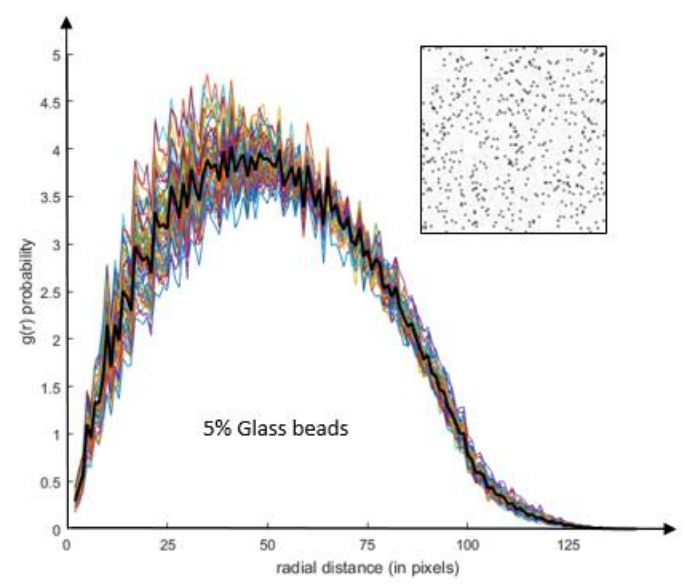



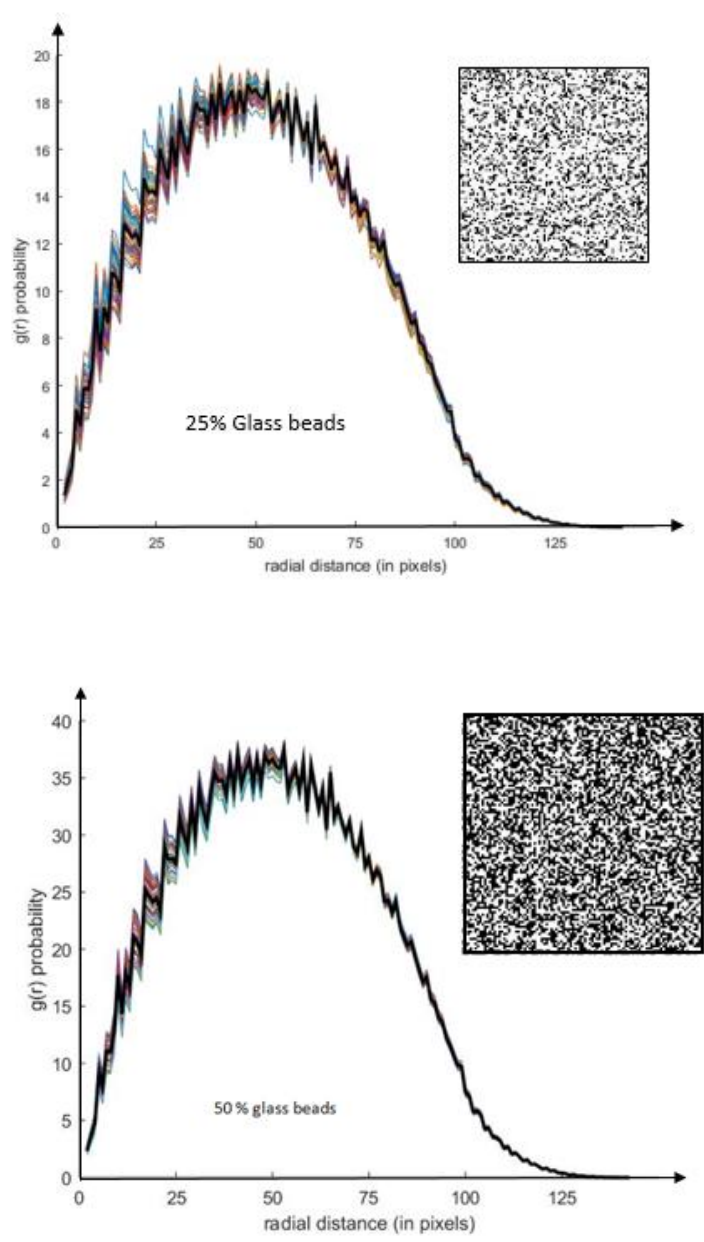

Figure 20: Three different populations have random probability distribution of particles (pixels) 5\%, 25\% and $50 \%$ particle contents.

Numerical distributions were generated and confirm that $5 \%$ inclusions will cause the highest scatter due to the spatial arrangement of inclusions. The figures show the radial distribution functions for three different random arrangements of beads.

\section{CONCLUSIONS}

The microstructure near the indentor tip has a predictable and consistent impact on the fracture toughness, this was concluded based on the following observations:

1. The fracture toughness changes in the experimental model system in which this is the only variable.
2. The 'kink' trend observed in the model system can be reproduced with a viscoelastic model that considers the effect of the nearest inclusion to the indentor probe

3. The trend in scatter with volume fraction of beads matches the probability of finding an inclusion near the indentor probe.

As a result, we propose a new formulation is required to obtain agreement between scratch and three-point fracture parameters by updating the $J$-integral formulation found in Akono et al.to capture the effect of local inclusions on the stress field generated by the indenter. Introducing random field theory into fracture resistance curves would allow us to extend the microscratching method to quantify fracture parameters of heterogeneous microstructures accurately.

\section{REFERENCES}

[1] Akono, AT. 2013. "Assessment of fracture properties and rate effects on fracture of materials by micro scratching application to gas shale." PhD dissertation, Massachusetts Institute of Technology. pp. 23-50.

[2] Akono, T. Ange, Randall X. Nicholes, Ulm F. Josef. 2012." Experimental determination of the fracture toughness via microscratch tests: Application to polymers, ceramics and metals." Material research society Journal, vol. 27, No. 2. Pp 485-493.

[3] Sanchez-Soto M., Pages P., Lacort T., Briceno K. 2007. Curing FTIR study and mechanical characterization of glass filled trifunctional epoxy composites. Composites science and Technology 67. pp 1974-1985.

[4] Erwin M. Wouterson, Freddy Y.C. Boey, Xiao $\mathrm{Hu}$, Shing-Chung Wong. 2005. "Specific properties and fracture toughness of syntactic foam: Effect of foam microstructures." Composites science and Technology 65. pp 1840-1850.

[5] Lee, J., and Yee, A.F.2000. "Fracture of glass bead/epoxy composites: on micro- 
mechanical deformations." Polymer 41. pp 8363-8373.

[6] Otsu, N.1979. "A Threshold Selection Method from Gray-Level Histograms," IEEE Transactions on Systems, Man, and Cybernetics, Vol. 9, No. 1, pp. 62-66.

[7] Anon." Standard test method for LinearElastic Plain-Strain Fracture Toughness KIC of Metallic Materials". ASTM E399-12. New York: Published January 2013.

[8] Zhonghua Li, Qiang C. 2003. Some simple formulas to predict the variation of stress intensity factors for mode I crack induced by near crack-tip inclusion. Journal of Engineering Fracture Mechanics. Volume 70. Pp. 581-587.

[9] Anderson.T.L. 2005. Fracture Mechanics fundamentals and applications. pp (257267). 